Pacific Journal of Mathematics

AMENABILITY AND KUNZE-STEIN PROPERTY FOR GROUPS 


\title{
AMENABILITY AND KUNZE-STEIN PROPERTY FOR GROUPS ACTING ON A TREE
}

\section{Claudio Nebbia}

\begin{abstract}
We characterize the amenable groups acting on a locally finite tree. In particular if the tree is homogeneous and the group $G$ acts transitively on the vertices then we prove that $G$ is amenable iff $G$ fixes one point of the boundary of the tree. Moreover we prove that a group $G$ which acts transitively on the vertices and on an open subset of the boundary is either amenable or a Kunze-Stein group.
\end{abstract}

1. Introduction and notations. Let $X$ be a locally finite tree, that is, a connected graph without circuits such that every vertex belongs to a finite set of edges. Let $V$ be the set of vertices and $E$ the set of edges. If $v_{1}$ and $v_{2}$ are in $V$, let $\left[v_{1}, v_{2}\right]$ be the unique geodesic connecting $v_{1}$ to $v_{2}$; the distance $d\left(v_{1}, v_{2}\right)$ is defined as the length of the geodesic $\left[v_{1}, v_{2}\right]$. Let $\operatorname{Aut}(X)$ be the locally compact group of all isometries of $X$ and, for $x \in V$, let $K_{x}$ be the stability subgroup of $x$; $K_{X}$ is a compact open subgroup of $\operatorname{Aut}(X)$. Let $\Omega$ be the boundary of the tree, that is, the set of equivalence classes of sequences of distinct vertices $\left\{s_{n}\right\}, n=0,1,2, \ldots$, such that $\left[s_{i}, s_{i+1}\right]$ is an edge for every $i=0,1,2, \ldots, n, \ldots$ (two such sequences are said to be equivalent if they have infinitely many common vertices). $\Omega$ is a compact metric space; every class of $\Omega$ is called an end of the tree. If $x_{0} \in V$ and $\omega_{0} \in \Omega$, there exists a unique geodesic $\left[x_{0}, \omega_{0}\right]$ from $x_{0}$ to $\omega_{0}$, that is, a unique sequence $\left\{s_{n}\right\}$ of distinct vertices $\left\{s_{0}, s_{1}, \ldots, s_{n}, \ldots\right\}$ in the class $\omega_{0}$ such that $s_{0}=x_{0}$. Hence $\Omega$ can also be regarded, as the set of infinite sequences starting from any fixed vertex $x_{0} \in V$.

In the same way, for $\omega_{1}, \omega_{2} \in \Omega$ with $\omega_{1} \neq \omega_{2}$, let $\left[\omega_{1}, \omega_{2}\right]$ be the unique geodesic joining $\omega_{1}$ to $\omega_{2} ;\left[\omega_{1}, \omega_{2}\right]$ is a line, that is, a sequence $\left\{s_{n}\right\}, n=0, \mp 1, \mp 2, \mp 3, \ldots$, of distinct vertices such that $\left[s_{i}, s_{i+1}\right]$ is an edge for every $i$. Conversely, every line is associated with a pair of ends of $X$. The reader is referred to $[2,3]$ for more details.

For $g \in \operatorname{Aut}(X)$, J. Tits has proved in [6] that one and only one of the following holds:

(1) There exists a vertex $v \in V$ such that $g(v)=v$ (in this case $g$ is called a rotation). 
(2) There exists an edge $[x, y]$ such that $g(x)=y$ and $g(y)=x(g$ is called an inversion).

(3) There exist a line $\left\{s_{n}\right\}$ and an integer $j \neq 0$ such that $g\left(s_{n}\right)=s_{n+j}$ for every $n$ ( $g$ is called a step $|j|$ translation on the line $C=\left\{s_{n}\right\}$. Moreover $\{j \mid=\min \{d(v, g(v)): v \in V\}$ and $C=\{v \in V: d(v, g(v))=$ $|j|\})$. Furthermore, J. Tits proved in [7] that a solvable fixed point free group of isometries of a tree leaves invariant an end or a pair of ends of $X$. But the stability subgroup of an end or a pair of ends, in general, is not solvable. In this paper we consider the larger class of amenable groups and we prove that, if $X$ is a locally finite tree and $G$ is a closed noncompact subgroup of $\operatorname{Aut}(X)$, then $G$ is amenable iff $G$ leaves invariant an end or a pair of ends of $X$. In particular, we deduce that a group $G$ which acts transitively on the vertices of a homogeneous tree of order $r>2$ (that is, a tree where every vertex belongs to $r$ edges) is amenable iff $G$ fixes one end. In this case we give another characterization of amenability, and we observe that $G$ acts transitively on an open subset of the boundary $\Omega$.

We recall that a locally compact group $G$ satisfies the "Kunze-Stein property" if $L^{p}(G)^{*} L^{2}(G) \subset L^{2}(G)$ for every $1<p<2$ (a group of this type will be called a "Kunze-Stein group"). A closed subgroup of $\operatorname{Aut}(X)$ ( $X$ is a homogeneous or semihomogeneous tree) which acts transitively on $\Omega$ is a Kunze-Stein group [5]. In $\$ 3$ below we show that if $G$ acts transitively on the vertices and on an open subset of $\Omega$ then $G$ is either amenable or a Kunze-Stein group. If no orbit of $G$ on $\Omega$ is open, then $G$ is not amenable and we conjecture that $G$ is not a Kunze-Stein group. This amounts to conjecture that, for groups acting transitively on the vertices, the Kunze-Stein property is equivalent to the fact that the group acts transitively on the tree boundary $\Omega$.

Finally, we prove this conjecture in the special case of a homogeneous tree of order three.

This work was done while the author was visiting the Department of Mathematics of the University "L. Pasteur" of Strasbourg, whose warm hospitality is acknowledged with pleasure.

We would like to thank the referee for his valuable suggestions and comments.

2. Amenable groups acting on a tree. We consider the following four properties:

$\left(P_{1}\right) G$ fixes one vertex.

$\left(P_{2}\right) G$ leaves invariant an edge (that is $\left.g([x, y])=[x, y]\right)$. 
$\left(P_{3}\right) G$ fixes one end of $X$.

$\left(P_{4}\right) G$ leaves invariant a pair of ends of $X$.

The main result of this section is the following theorem.

THEOREM 1. Let $X$ be a locally finite tree and $G$ a closed subgroup of $\operatorname{Aut}(X)$. Then $G$ is amenable if and only if $G$ has property $\left(P_{i}\right)$ for some $i(i=1,2,3,4)$.

Proof. Let $G$ be a closed amenable subgroup of $\operatorname{Aut}(X)$. Let us prove that there exists $i(1 \leq i \leq 4)$ such that $G$ has property $\left(P_{i}\right)$. Observe that if $\phi$ and $\psi$ are translations on the lines $C_{1}$ and $C_{2}$, respectively, and $C_{1} \cap C_{2}=\varnothing$ then the subgroup $\langle\phi, \psi\rangle$ generated by $\phi$ and $\psi$ is a discrete subgroup of $\operatorname{Aut}(X)$ isomorphic to the free group with two generators. This is a consequence of the following claim: $w\left(C_{1}\right) \cap$ $C_{1}=\varnothing$ for every reduced word $w$ in $\phi$ and $\psi$ such that $w \notin\langle\phi\rangle$, the subgroup generated by $\phi$. Indeed this implies that, for $x \in C_{1}$, $w(x) \neq x$ for every reduced word in $\phi$ and $\psi$. A fortiori, $w \neq 1$ and $\langle\phi, \psi\rangle \cap K_{x}=\{1\}$; this means that $\langle\phi, \psi\rangle$ is a discrete subgroup of $\operatorname{Aut}(X)$ isomorphic to the free group with two generators. We prove now the claim. Let $\gamma_{1}, \gamma_{2}$ be two lines such that $\gamma_{1} \cap \gamma_{2}=\varnothing$; we define $\left[\gamma_{1}, \gamma_{2}\right]=\left[x_{0}, y_{0}\right]$ where $x_{0} \in \gamma_{1}, y_{0} \in \gamma_{2}$ and $d\left(x_{0}, y_{0}\right)=d\left(\gamma_{1}, \gamma_{2}\right)=$ $\min \left\{d(x, y): x \in \gamma_{1}, y \in \gamma_{2}\right\}$.

Let $\gamma$ be a line such that $\gamma \cap C_{1}=\gamma \cap C_{2}=\varnothing$; it is easy to see that $\left[\gamma, C_{1}\right] \subset\left[\gamma, C_{2}\right]$ implies that $\psi^{m}(\gamma) \cap C_{1}=\psi^{m}(\gamma) \cap C_{2}=$ $\varnothing$ and $\left[\psi^{m}(\gamma), C_{2}\right] \subset\left[\psi^{m}(\gamma), C_{1}\right]$ for every $m \neq 0$. Similarly, if $\left[\gamma, C_{2}\right] \subset\left[\gamma, C_{1}\right]$, then $\phi^{m}(\gamma) \cap C_{1}=\phi^{m}(\gamma) \cap C_{2}=\varnothing$ and $\left[\phi^{m}(\gamma), C_{1}\right] \subset$ $\left[\phi^{m}(\gamma), C_{2}\right]$.

Since, for every $m \neq 0, \phi^{m}\left(C_{1}\right)=C_{1}, \psi^{m}\left(C_{1}\right) \cap C_{1}=\psi^{m}\left(C_{1}\right) \cap C_{2}=$ $\varnothing$ and $\left[\psi^{m}\left(C_{1}\right), C_{2}\right] \subset\left[\psi^{m}\left(C_{1}\right), C_{1}\right]$ it follows that $w\left(C_{1}\right) \cap C_{1}=$ $w\left(C_{1}\right) \cap C_{2}=\varnothing$ for $w=\phi^{j_{1}} \psi^{i_{1}} \phi^{j_{2}} \psi^{i_{2}} \ldots \phi^{j_{n}} \psi^{i_{n}} \phi^{j}$ with $j_{2}, \ldots, j_{n}, i_{1}$, $i_{2}, \ldots, i_{n}$ nonzero integers, $n>0$. This proves the claim. Hence, if $\phi, \psi \in G$ then $C_{1} \cap C_{2} \neq \varnothing$. We can suppose that there exists a translation $\phi \in G$ on $C$, otherwise $G$ satisfies $\left(P_{1}\right)$ or $\left(P_{2}\right)$ or $\left(P_{3}\right)$ [6, Prop. 3.4]. For every $g \in G, g \phi g^{-1}$ is a translation on the line $g(C)$, and thus by the argument above $C \cap g(C) \neq \varnothing$.

But if $C \cap g(C)$ is finite for a $g \in G$, then there exists $j$ such that $\phi^{j}(C \cap g(C)) \cap(C \cap g(C))=\varnothing$, and thus, since $g(C) \cap\left\{\phi^{j}(g(C))\right\} \neq \varnothing$ would force existence of a circuit joining $C \cap g(C)$ and $\phi^{j}(g(C))$, $g(C) \cap\left\{\phi^{j}(g(C))\right\}=\varnothing$ which is a contradiction. Thus $C \cap g(C)$ is infinite for every $g \in G$. We denote by $\omega_{1}, \omega_{2}$ the two ends of 
C. Then $g \omega_{1}$ and $g \omega_{2}$ are the two ends of $g(C)$. If $C=g(C)$, then $g \omega_{1}=\omega_{1}$ and $g \omega_{2}=\omega_{2}$ or $g \omega_{1}=\omega_{2}$ and $g \omega_{2}=\omega_{1}$. If $C \neq g(C)$ then the set: $A_{g}=\left\{\omega_{1}, \omega_{2}\right\} \cap\left\{g \omega_{1}, g \omega_{2}\right\}$ is a singleton because $C \cap g(C)$ is infinite. We prove now that $A_{g}=A_{h}$ for every $g$ and $h$ in $G$ such that $g(C) \neq C \neq h(C)$. To prove this, we observe that if $g(C) \neq C$ and $A_{g}=\left\{\omega_{2}\right\}$ then $g \omega_{2}=\omega_{2}$. Indeed let $g \omega_{2} \neq \omega_{2}$ and $A_{g}=\left\{\omega_{2}\right\}$, then $g \omega_{1}=\omega_{2}$ and $g \omega_{2} \neq \omega_{1}$; therefore $g g \omega_{2}=\omega_{1}$ (otherwise $g g \omega_{2} \neq \omega_{2}$ and $A_{g g}=\varnothing$ which is impossible). This means that $g$ is a cyclic permutation of the set $\left\{\omega_{1}, \omega_{2}, g \omega_{2}\right\}$. We consider the element $\phi g \in G$; it follows that $\phi g(C) \neq C$ and $\phi g \omega_{1}=\phi \omega_{2}=$ $\omega_{2}, \phi g \omega_{2} \neq \omega_{2}$. The reasoning above implies that $\phi g$ is a cyclic permutation of the set $\left\{\omega_{1}, \omega_{2}, \phi g \omega_{2}\right\}$. In particular, $\phi g\left(\phi g \omega_{2}\right)=\omega_{1}$, that is, $\phi g \omega_{2}=g^{-1} \phi^{-1} \omega_{1}=g \omega_{2}$. This is a contradiction because $\phi$ is a translation on $C$ and $\phi \omega=\omega$ iff $\omega=\omega_{1}$ or $\omega=\omega_{2}$. This proves that $g \omega_{2}=\omega_{2}$.

The proof of the fact that $A_{g}=\left\{\omega_{1}\right\}$ implies that $g \omega_{1}=\omega_{1}$ is similar. We suppose now that there exist $g$ and $h$ in $G$ such that $A_{g}=\left\{\omega_{1}\right\}$ and $A_{h}=\left\{\omega_{2}\right\}$, then $g h \omega_{2} \neq \omega_{2}$ and $g h \omega_{1} \neq \omega_{1}$ which implies that $g h(C)=C$ and so $g h \omega_{2}=\omega_{1}$ and $g \omega_{2}=\omega_{1}$, contrary to the assumption that $g \omega_{1}=\omega_{1}$. Thus $A_{g}=A_{h}$ for every $g$ and $h$ such that $g(C) \neq C \neq h(C)$, say $A_{g}=\left\{\omega_{2}\right\}$. Therefore for any given $g$ in $G$, we have the following two mutually exclusive possibilities: (1) $g \omega_{2}=\omega_{2} ;(2) g \omega_{1}=\omega_{2}$ and $g \omega_{2}=\omega_{1}$. Hence $G$ has property $\left(P_{3}\right)$ or $\left(P_{4}\right)$ because it is easy to see that if there exists an isometry of type (2) then $g \omega_{2}=\omega_{2}$ implies $g \omega_{1}=\omega_{1}$. Conversely, we now prove that every group which has property $\left(P_{i}\right), i=1,2,3,4$, is amenable. If $G$ is the stability subgroup of a vertex or of an edge then $G$ is compact because $X$ is locally finite. Now, let $G$ be the stability subgroup of an end $\omega_{0} \in \Omega$. Let $\left\{s_{0}, s_{1}, \ldots, s_{n}, \ldots\right\}$ be the geodesic from $s_{0}$ to $\omega_{0}$ and $B$ the group of all isometries $b$ such that $b\left(s_{n}\right)=s_{n}$ for $n$ sufficiently large. Then $B \subset G$ and $B=\bigcup_{n=0}^{\infty} B_{n}$ where $B_{n}=G \cap K_{s_{n}}$; since $B_{n}$ is compact open in $G$ the group $B$ is a closed amenable subgroup of $\operatorname{Aut}(X)$, open in $G, B$ is the subset of rotations of $G$ : indeed, if $g \in G$ fixes a vertex $v$, it also fixes the geodesic $\left[v, \omega_{0}\right]$, hence $g \in B$. Moreover, by definition, $G$ contains no inversions. Therefore, if $G \neq B$ there exists a step $j$ translation $\phi$ on $C=\left\{s_{n}\right\}$ and we can choose $\phi$ in such a way that $j$ is smallest possible. In particular if $G$ contains translations of step $j^{\prime}$ then $j^{\prime}$ is a multiple of $j$. Hence $G=\langle\phi\rangle B$ where $\langle\phi\rangle$ is the group generated by $\phi$. Because $B \cap\langle\phi\rangle=\{1\}$ and $B$ is a normal amenable subgroup of $G$, it follows that $G$ is amenable. Finally, 
let $\omega_{1}, \omega_{2} \in \Omega$ with $\omega_{1} \neq \omega_{2}$ and denote by $C=\left\{s_{n}\right\}, n \in \mathbf{Z}$, the geodesic joining $\omega_{1}$ to $\omega_{2}$, and by $G$ the group which leaves invariant the set $\left\{\omega_{1}, \omega_{2}\right\}$. Let $K_{0}=\left\{h \in G: h\left(s_{n}\right)=s_{n}\right.$ for every $\left.n\right\}$; by [6, Proposition 3.4\} we can suppose that there exists a step $j$ translation $\phi$ on $C$ and $j$ is smallest possible. Then $K_{0}\langle\phi\rangle$ is an amenable closed subgroup of $G\left(K_{0}\right.$ is compact normal in $\left.K_{0}\langle\phi\rangle\right)$. It is easy to see that $K_{0}\langle\phi\rangle$ is the set of isometries of $G$ such that $g \omega_{1}=\omega_{1}$ and $g \omega_{2}=\omega_{2}$. Hence, if $G \neq K_{0}\langle\phi\rangle$ then $K_{0}\langle\phi\rangle$ is a closed normal amenable subgroup of index 2 in $G$. Then $G$ is amenable and the theorem is proved.

REMarks. 1. The first part of Theorem 1 holds for a general tree; in other words a closed amenable subgroup of $\operatorname{Aut}(X)$, where $X$ is a general tree, has property $\left(P_{i}\right)$ for some $i=1, \ldots, 4$. But the converse is not true for a general tree. Indeed, let $X_{0}$ be the tree with vertex set $V=\mathrm{N} \cup\{\infty\}$ and edge set $E=\{\{n, \infty\}: n \in \mathbf{N}\} . X_{0}$ is not a locally finite tree and $\operatorname{Aut}\left(X_{0}\right)=K_{\infty} \simeq S(\mathbf{N})$, the group of all permutations of $\mathbf{N}$, is not amenable.

2. We shall say that a vertex $v$ is of homogeneity $l$ if $v$ belongs to exactly $l$ edges. A semihomogeneous tree $X_{l, q}$ is a tree such that every vertex is of homogeneity $l$ or $q$ and two adjacent vertices are of homogeneity $l$ and $q$, respectively. Let $S_{l}$ [respectively $S_{q}$ ] be the subset of $X_{l, q}$ of vertices of homogeneity $l$ [respectively $q$ ]. If $l=q=$ $r$, then $X_{l, q}$ is a homogeneous tree of order $r$. Let $X$ be a homogeneous tree and $G$ be a closed subgroup of $\operatorname{Aut}(X)$ acting transitively on the vertices, or alternatively let $X=X_{l, q}$ and $G$ be a closed subgroup of $\operatorname{Aut}\left(X_{l, q}\right)$ acting transitively on $S_{l}$. It follows from Theorem 1 that $G$ is amenable iff $G$ fixes one end. In fact, if $G$ has property $\left(P_{1}\right),\left(P_{2}\right)$ or $\left(P_{4}\right)$ then, for some vertex $v$, the orbit $G v$ is either finite or contained in a line.

3. We can deduce from the proof of Theorem 1 that a closed nonamenable subgroup of $\operatorname{Aut}(X)$ contains a discrete subgroup isomorphic to the free group with two generators. Indeed if $G$ is not amenable, Proposition 3.4 of [6] implies that $G$ contains a translation $\phi$ on $C$. The proof of Theorem 1 shows that if $g(C) \cap C \neq \varnothing$ for every $g$ in $G$, then $G$ is amenable. Therefore there exists $g \in G$ such that $g(C) \cap C=\varnothing$, and so the subgroup of $G$ generated by $\phi$ and $g \phi g^{-1}$ is a discrete group isomorphic to the free group with two generators.

4. In [6] J. Tits proved that a solvable subgroup of $\operatorname{Aut}(X)$ has property $\left(P_{1}\right),\left(P_{3}\right)$ or $\left(P_{4}\right)$; but the vice-versa is not true. For example, the stabilizer of a vertex or an end, and the stabilizer of an edge or 
a pair of ends of a homogeneous or semihomogeneous tree are not solvable. Since this group contains subgroups of type $K_{C}=\{g: g(x)=$ $x$ for every $x \in C\}$ where $C=\left\{s_{n}: n \in Z\right\}$ is a line, it is enough to prove that $K_{C}$ is not solvable. Let $E_{m}$ be the following set:

$$
E_{m}=\left\{y \in X: d\left(y, s_{0}\right)=m \text { and }\left[s_{0}, y\right] \cap C=\left\{s_{0}\right\}\right\}
$$

for $m>0$, and $K_{m}=\left\{g \in K_{C}: g(y)=y\right.$ for every $\left.y \in E_{m}\right\}$.

$K_{m}$ is a closed normal subgroup of $K_{C}$ and $K_{C} / K_{m}$ is isomorphic to $S\left(E_{m}\right)$, the group of all permutations of $E_{m}$.

When $m$ is sufficiently large, $\left|E_{m}\right| \geq 5$ and $S\left(E_{m}\right)$ is not solvable. This implies that $K_{C}$ is not solvable.

Now we give another characterization of amenability for groups acting transitively on a homogeneous tree $X$. For $x \in V$ and for an integer $n \geq 0$ let $S_{n}^{x}=\{y \in V: d(x, y)=n\}$.

We define the following "local property $(*)$ ":

(*) For every $x \in V$ there exists $\tilde{x} \in V$ with $d(x, \tilde{x})=1$ such that:

(1) $G \cap K_{x} \subsetneq G \cap K_{\tilde{x}}$

(2) $G \cap K_{x}$ acts transitively on $S_{1}^{x} \backslash\{\tilde{x}\}$.

THEOREM 2. Let $X$ be a homogeneous tree of order $r>2$ and $G$ a closed subgroup of $\operatorname{Aut}(X)$ which acts transitively on the vertices, then the following are equivalent.

(a) $G$ is amenable.

(b) $G$ fixes one end of $X$.

(c) G has property (*).

Proof. As observed in Remark $2,(a) \Longleftrightarrow$ (b) follows from Theorem 1. If (*) holds then for every $x \in V$ there exists a unique $y=\tilde{x}$ such that $G \cap K_{x} \varsubsetneqq G \cap K_{\tilde{x}}$; in particular $x \neq \tilde{\tilde{x}}$. This implies that there exists a unique $G$-invariant path $C_{x}=\left\{s_{0}, s_{1} \ldots, s_{n}, \ldots\right\}$ starting at $s_{0}=x$ such that $s_{i+1}=\hat{s}_{i}$ for every $i$. To prove that $G$ fixes one end of $X$ it is enough to prove that $C_{x} \cap C_{y}$ is infinite for every $x, y \in V$.

If $x, y \in V$ and $d(x, y)=1$ then $C_{x} \cap C_{y}$ is infinite because $\tilde{x}=y$ or $\tilde{y}=x$. In fact, let $W_{x}=S_{1}^{x} \backslash\{\tilde{x}\}$. Because $g(\tilde{x})=[g(x)]^{\sim}$ for every $g \in G$ and $x \in V$, we have the following two mutually exclusive possibilities: (1) $\tilde{v}=x$ for every $v \in W_{x}$ and for every $x \in V$; (2) $\tilde{v} \neq x$ for every $v \in W_{x}$ and for every $x \in V$. But if $x \in V$ satisfies (2) then $\tilde{v}$ satisfies (1) because $\tilde{\tilde{v}} \neq v$ (by property (*)) and $v \in W_{\tilde{v}}$. This means that every vertex $x \in V$ satisfies (1). In particular $y=\tilde{x}$ or $x=\tilde{y}$. By induction on the distance $d(x, y)$ we have that $C_{x} \cap C_{y}$ is infinite for every $x, y \in V$, and (c) $\Rightarrow(\mathbf{b})$. Conversely, we 
now show that $(b) \Rightarrow(c)$. Let $G$ be a closed amenable subgroup of $\operatorname{Aut}(X)$ which acts transitively on the vertices. Let $s_{0} \in V$. Then $G$ must fix some end $\omega=\left[\left\{s_{0}, s_{1}, s_{2}, \ldots\right\}\right]$. We claim (*) holds with $\tilde{s}_{0}=s_{1}$. The fact that $G \cap K_{s_{0}} \subset G \cap K_{s_{1}}$ follows from the fact that $G$ fixes $\omega$. Suppose $u, v \in S_{1}^{s_{0}} \backslash\left\{s_{1}\right\}$. There exists $g \in G$ such that $g(u)=v$ because $G$ acts transitively on the vertices. Since $g$ fixes the end $\omega=\left[\left\{u, s_{0}, s_{1}, \ldots\right\}\right]=\left[\left\{v, s_{0}, s_{1}, \ldots\right\}\right], g \in K_{s_{0}}$; proving (2) of (*). The argument above implies that $G \cap K_{s_{1}}$ acts transitively on $S_{1}^{s_{t}} \backslash\left\{s_{2}\right\} \ni s_{0}$. If the tree is homogeneous of order $>2$, then there exists an element $w \in S_{1}^{s_{1}} \backslash\left\{s_{0}, s_{2}\right\}$, this implies that there exists $k \in G \cap K_{s_{1}}$ such that $k\left(s_{0}\right)=w \neq s_{0}$. This means that $G \cap K_{s_{0}} \neq G \cap K_{s_{1}}$. Since $G$ acts transitively on the vertices, property $(*)$ holds.

REMARK 5. If $G$ and $\phi$ are as in the proof of Theorem 1 then $G=\langle\phi\rangle K_{0}\langle\phi\rangle$. Since $G\left(s_{0}\right)=V$ and $\langle\phi\rangle\left(s_{0}\right)=\left\{s_{n}: n \in \mathbf{Z}\right\}$, it follows that, for every $v \in V$ with $d\left(s_{0}, v\right)=n$ and $v \neq s_{-n}$, there exists $k \in K_{0}$ such that $k\left(s_{-n}\right)=v$. In other words, $G$ acts transitively on an open subset of $\Omega$, in fact on $\Omega \backslash\left\{\omega_{0}\right\}$ where $\omega_{0}$ is the fixed end of $X$.

3. Groups acting transitively on an open subset of $\Omega$. In this section we prove the following result:

Theorem 3. Let $X$ be a homogeneous tree and let $G$ be a closed subgroup of $\operatorname{Aut}(X)$ which acts transitively on the vertices and on an open subset of $\Omega$. Then either $G$ fixes one end of $X$ (i.e. $G$ is amenable) or $G$ acts transitively on $\Omega$ (i.e. $G$ is a Kunze-Stein group).

Proof. It is enough to prove that there exists $\omega_{0} \in \Omega$ such that $G$ acts transitively on $\Omega \backslash\left\{\omega_{0}\right\}$; then the theorem follows from Theorem 2 and [5].

It follows, by [6], that there exists a translation $\phi \in G$ on a line $C=\left\{s_{n}\right\}$. In this proof we realize $\Omega$ as the set of all infinite paths issued from $s_{0}$. Then the sets: $E(x)=\left\{\left\{t_{n}\right\} \in \Omega: t_{j}=x\right\}$ with $x \in V$ and $d\left(s_{0}, x\right)=j$ form a basis for the topology of $\Omega$. Also, we observe that, for $\omega_{0} \in \Omega$, the orbit $G \omega_{0}$ is open iff $\left(G \cap K_{S_{0}}\right) \omega_{0}$ is open: this follows from Baire's theorem and the fact that $G /\left(G \cap K_{s_{0}}\right)$ is countable.

In particular $G \cap K_{S_{0}}$ acts transitively on a set $E(x)$. Let $h \in G$ such that $h\left(s_{0}\right)=x$. Then $h \phi h^{-1}$ is a translation and, without loss of generality, we can suppose that $\phi$ is a translation on $C=\left\{s_{n}\right\}$ and $G \cap K_{s_{0}}$ acts transitively on $\mathcal{C} E\left(s_{-1}\right)$. Let $\omega_{0}=\left\{s_{0}, s_{-1}, s_{-2}, \ldots\right\}$.

Since $G$ contains the translation $\phi$, then $G$ acts transitively on $\complement E\left(s_{-n}\right)$ for every $n \geq 0$, hence on $\Omega \backslash\left\{\omega_{0}\right\}=\bigcup_{n \geq 0} \mathcal{\complement} E\left(s_{-n}\right)$. 
REMARK 6. The same statement holds, with the same proof, for semihomogeneous tree $X_{l, q}$ and groups acting transitively on $S_{l}$.

Theorem 3 means that if there exists an open orbit of $G$ on $\Omega$ then $G$ is either amenable or a Kunze-Stein group. What happens if no orbit is open? As observed in Remark 5, $G$ is not amenable.

We conjecture that $G$ is not a Kunze-Stein group. However, we are able to prove this conjecture only in the special case of a homogeneous tree of order three.

Denote by $\Gamma$ the simply transitive subgroup of $\operatorname{Aut}(X)$ isomorphic with $\mathbf{Z}_{2} * \mathbf{Z}_{2} * \cdots \mathbf{Z}_{2} r$-times considered in $[1,4]$.

LEMMA 1. Let $G$ act transitively on the vertices of a homogeneous tree. Then $G$ contains a step one translation iff $G \neq \Gamma$.

Proof. By [1, 4] it is enough to prove that if $G \cap K_{x} \neq\{1\}$ then there exists a step one translation in $G$.

If $G \cap K_{x} \neq\{1\}$ then there exists $k \in G \cap K_{x}$ and $a \neq b$ with $a, b \in S_{1}^{x}$ such that $k(a)=b$; this follows from the fact that $G$ acts transitively on the vertices. Choose $g$ in $G$ such that $g(x)=b$. Then $g$ is either a step one translation or an inversion. If $g$ is not a step one translation then $g(b)=x$; hence $g k$ is a step one translation because $g k(a)=x$ and $g k(x)=b$.

In particular this translation exists if $G$ is not discrete or if $G$ is amenable.

Lemma 2. Let $G$ be a closed subgroup of $\operatorname{Aut}(X)$, let $\phi \in G$ be a translation on a line $C=\left\{s_{n}\right\}$ and $\omega$ an end of $C$. If the orbit $\left(G \cap K_{s_{0}}\right) \omega$ is finite, then $G$ is not a Kunze-Stein group.

Proof. Let $K^{\prime}$ be the stabilizer of $\omega$ in $G \cap K_{s_{0}}$. Let $H$ be the subgroup of $G$ generated by $K^{\prime}$ and $\phi$. By assumptions $\left(G \cap K_{s_{0}}\right) / K^{\prime}$ is finite, therefore $K^{\prime}$ is open in $G \cap K_{s_{0}}$, hence in $G$. This implies that $H$ is open in $G$ and closed in $\operatorname{Aut}(X)$. By Theorem $1, H$ is amenable.

Therefore $H$ is an open amenable noncompact subgroup of $G$. The result now follows from the facts that amenable noncompact groups are not Kunze-Stein groups and that every open subgroup of a KunzeStein group is a Kunze-Stein group.

Proposition. If $G$ is a closed Kunze-Stein group which acts transitively on the vertices of a homogeneous tree of order three, then $G$ acts transitively on $\Omega$. 
Proof. Let $G$ be a closed Kunze-Stein subgroup of $\operatorname{Aut}(X)$ which acts transitively on the vertices of $X$. Then $G$ contains a step one translation $\phi$ on a line $C=\left\{s_{n}\right\}$ and $\left(G \cap K_{s_{0}}\right) \omega_{0}$ is infinite (here $\left.\omega_{0}=\left\{s_{0}, s_{1}, \ldots\right\} \in \Omega\right)$. This follows from Lemma 1 and Lemma 2 .

As $\omega_{0}$ is an accumulation point of the orbit $\left(G \cap K_{s_{0}}\right) \omega_{0}$, then there exists a sequence $n_{m} \rightarrow+\infty$ and a sequence $k_{n_{m}} \in G \cap K_{s_{0}}$ such that $k_{n_{m}}\left(s_{n_{m}}\right)=s_{n_{m}}$ but $k_{n_{m}}\left(s_{n_{m}+1}\right) \neq s_{n_{m}+1}$. Now the rotations $\phi^{i} k_{n_{m}} \phi^{-i}$ in $G \cap K_{s_{0}}$, for $i=0,1, \ldots, n_{m}$, yield a sequence $\tilde{k}_{n} \in G \cap K_{s_{0}}$ such that $\tilde{k}_{n}\left(s_{n}\right)=s_{n}$ but $\hat{k}_{n}\left(s_{n+1}\right) \neq s_{n+1}$ for every $n \geq 0$. The same argument applies to every point $\omega^{\prime}=k \omega_{0}$ of the orbit $\left(G \cap K_{s_{0}}\right) \omega_{0}$ by replacing $C$ with $k(C)$ and $\phi$ with the step one translation $k \phi k^{-1}$ on $k(C)$. Since $G \cap K_{s_{0}}$ is compact, the orbit $\left(G \cap K_{s_{0}}\right) \omega_{0}$ is closed. If $r=3$, the argument above shows that $\omega_{0}$ is an interior point of this orbit. Thus the orbit is open and, by Theorem $3, G$ acts transitively on $\Omega$.

REMARK 7. Finally, we provide an example of a nondiscrete subgroup of $\operatorname{Aut}(X)$ acting transitively on the vertices and such that no orbit on $\Omega$ is open. Let $E_{1}$ be a subset of edges of $X$ such that there are no two adjacent edges in $E_{1}$ and every vertex belongs to exactly one edge of $E_{1}$. Let $G_{1}$ be the stability group of $E_{1}$; then it is easy to see that $G_{1}$ is a closed subgroup of $\operatorname{Aut}(X)$ which acts transitively on the vertices but $G_{1}$ is not discrete because $G_{1} \cap K_{x}$ is infinite.

If $g \in G_{1}$ and $\omega \in \Omega$ is a path which contains a finite number of edges of $E_{1}$ then also $g \omega$ contains a finite number of edges of $E_{1}$, in particular no orbit of $G$ on $\Omega$ is open.

Observe, also that $G_{1}$ is not amenable, hence it does not satisfy the local property (*) of Theorem 2 . On the other hand, it satisfies the following property $(* *)$ :

(**) For every $x \in V$ there exists $\tilde{x} \in V$ with $d(x, \tilde{x})=1$ and such that:

(1) $G \cap K_{x}=G \cap K_{\tilde{x}}$

(2) $G \cap K_{x}$ acts transitively on $S_{1}^{x} \backslash\{\tilde{x}\}$.

In this case, if $y=\tilde{x}$ then $\tilde{y}=x$.

\section{REFERENCES}

[1] W. Betori and M. Pagliacci, Harmonic analysis for groups acting on trees, Boll. Un. Mat. Ital., 3-B (1984), 333-349.

[2] P. Cartier, Geométrie et analyse sur les arbres, Sém. Bourbaki 1971/72 407, Lecture Notes in Math. 317, Springer-Verlag, 123-140. 
[3] P. Cartier, Fonctions harmoniques sur un arbres, Symposia Math., 9 (1972), 203-270.

[4] F. Choucroun, Groupes operant simplement transitivement sur un arbre homogène et plongements dans $\mathrm{PGL}_{2}(K), \mathrm{C}$. R. Acad. Sci. Paris, 298 (1984), 313-315.

[5] C. Nebbia, Groups of isometries of a tree and the Kunze-Stein phenomenon, Pacific J. Math., 133 (1988), 141-149.

[6] J. Tits, Sur le groupe des automorphismes d'un arbre, Essays on topology and related topics, Mémoires dédiés a G. de Rham, Springer-Verlag 1970, 188-211.

[7] J. Tits, $A$ "theorem of Lie-Kolchin" for trees, Contributions to Algebra, A Collection of Papers Dedicated to Ellis Kolchin, Academic Press 1977, 377-388.

Received November 24, 1986 and in revised form November 4, 1987. This work was partially supported by G.N.A.F.A. of the C.N.R., Italy.

UNIVERSITÀ DEGLi STUdi di ROMA "LA SAPIENZA"

Città Universitaria-00187 Roma, Italy 


\section{PACIFIC JOURNAL OF MATHEMATICS \\ EDITORS}

\author{
V. S. VARADARAJAN \\ (Managing Editor) \\ University of California \\ Los Angeles, CA 90024 \\ HERBERT CLEMENS \\ University of Utah \\ Salt Lake City, UT 84112 \\ R. FINN \\ Stanford University \\ Stanford, CA 94305
}

\author{
HERMANN FLASCHKA \\ University of Arizona \\ Tucson, AZ 85721
}

vAUGHAN F. R. JONES

University of California

Berkeley, CA 94720

STEVENKERCKH OFF

Stanford University

Stanford, CA 94305

\author{
ROBION KIRBY \\ University of California \\ Berkeley, CA 94720 \\ C. C. MOORE \\ University of California \\ Berkeley, CA 94720 \\ HAROLD T ARK
}

University of California, San Diego La Jolla, CA 92093

\section{ASSOCIATE EDITORS}
R. ARENS
E. F. BECKENBACH
B. H. NEUMANN
F. WOLF
K. YOSHIDA

(1906-1982)

\section{SUPPORTING INSTITUTIONS}

\author{
UNIVERSITY OF ARIZONA \\ UNIVERSITY OF BRITISH COLUMBIA \\ CALIFORNIA INSTITUTE OF TECHNOLOGY \\ UNIVERSITY OF CALIFORNIA \\ MONTANA STATE UNIVERSITY \\ UNIVERSITY OF NEVADA, RENO \\ NEW MEXICO STATE UNIVERSITY \\ OREGON STATE UNIVERSITY
}

\author{
UNIVERSITY OF OREGON \\ UNIVERSITY OF SOUTHERN CALIFORNIA \\ STANFORD UNIVERSITY \\ UNIVERSITY OF HAWAII \\ UNIVERSITY OF TOKYO \\ UNIVERSITY OF UTAH \\ WASHINGTON STATE UNIVERSITY \\ UNIVERSITY OF WASHINGTON
}

The Supporting Institutions listed above contribute to the cost of publication of this Journal, but they are not owners or publishers and have no responsibility for its content or policies.

Mathematical papers intended for publication in the Pacific Journal of Mathematics should be in typed form or offset-reproduced (not dittoed), double spaced with large margins. Please do not use built up fractions in the text of the manuscript. However, you may use them in the displayed equations. Underline Greek letters in red, German in green, and script in blue. The first paragraph must be capable of being used separately as a synopsis of the entire paper. In particular it should contain no bibliographic references. Please propose a heading for the odd numbered pages of less than 35 characters. Manuscripts, in triplicate, may be sent to any one of the editors. Please classify according to the scheme of Math. Reviews, Index to Vol. 39. Supply name and address of author to whom proofs should be sent. All other communications should be addressed to the managing editor, or Elaine Barth, University of California, Los Angeles, California 90024.

There are page-charges associated with articles appearing in the Pacific Journal of Mathematics. These charges are expected to be paid by the author's University, Government Agency or Company. If the author or authors do not have access to such Institutional support these charges are waived. Single authors will receive 50 free reprints; joint authors will receive a total of 100 free reprints. Additional copies may be obtained at cost in multiples of 50 .

The Pacific Journal of Mathematics is issued monthly as of January 1966. Regular subscription rate: $\$ 190.00$ a year (5 Vols., 10 issues). Special rate: $\$ 95.00$ a year to individual members of supporting institutions.

Subscriptions, orders for numbers issued in the last three calendar years, and changes of address should be sent to Pacific Journal of Mathematics, P.O. Box 969, Carmel Valley, CA 93924, U.S.A. Old back numbers obtainable from Kraus Periodicals Co., Route 100, Millwood, NY 10546.

The Pacific Journal of Mathematics at P.O. Box 969, Carmel Valley, CA 93924 (ISSN 0030-8730) publishes 5 volumes per year. Application to mail at Second-class postage rates is pending at Carmel Valley, California, and additional mailing offices. Postmaster: send address changes to Pacific Journal of Mathematics, P.O. Box 969, Carmel Valley, CA 93924.

\section{PUBLISHED BY PACIFIC JOURNAL OF MATHEMATICS, A NON-PROFIT CORPORATION} Copyright (C) 1988 by Pacific Journal of Mathematics 


\section{Pacific Journal of Mathematics}

Vol. 135, No. $2 \quad$ October, 1988

Waleed A. Al-Salam and Mourad Ismail, $q$-beta integrals and the

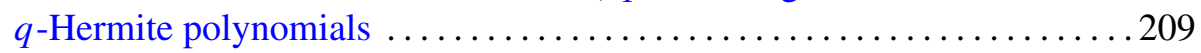

Johnny E. Brown, On the Ilieff-Sendov conjecture $\ldots \ldots \ldots \ldots \ldots \ldots \ldots 223$

Lawrence Jay Corwin and Frederick Paul Greenleaf, Spectrum and

multiplicities for restrictions of unitary representations in nilpotent Lie

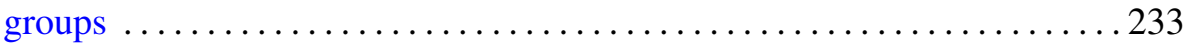

Robert Jay Daverman, 1-dimensional phenomena in cell-like mappings on 3-manifolds .......................................... 269

P. D. T. A. Elliott, A localized Erdős-Wintner theorem .............. 287

Richard John Gardner, Relative width measures and the plank problem . . . 299

F. Garibay, Peter Abraham Greenberg, L. Reséndis and Juan José

Rivaud, The geometry of sum-preserving permutations ............313

Shanyu Ji, Uniqueness problem without multiplicities in value distribution

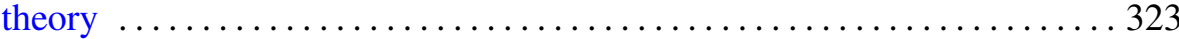

Igal Megory-Cohen, Finite-dimensional representation of classical

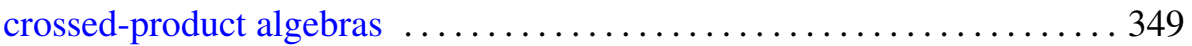

Mirko Navara, Pavel Pták and Vladimír Rogalewicz, Enlargements of

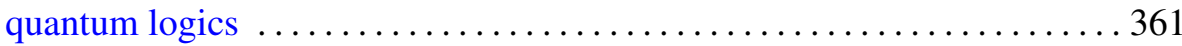

Claudio Nebbia, Amenability and Kunze-Stein property for groups acting

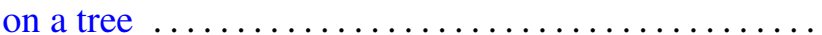

Chull Park and David Lee Skoug, A simple formula for conditional Wiener

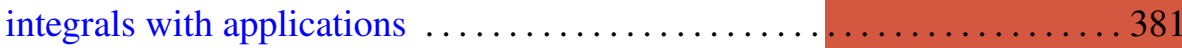

Ronald Scott Irving and Brad Shelton, Correction to: "Loewy series and simple projective modules in the category $\mathrm{O}_{s} " \ldots .$.

Robert Tijdeman and Lian Xiang Wang, Correction to: "Sums of products

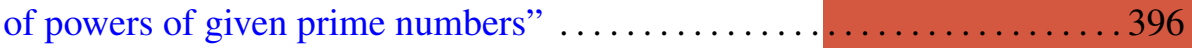

